\title{
BMJ Open Cohort profile: the Martinique Cancer Registry and the quality of life prostate cancer cohort (QoL Prostate-MQ): challenges and prospects for reducing disparities in the Caribbean
}

To cite: Joachim C, VeroniqueBaudin J, Almont T, et al. Cohort profile: the Martinique Cancer Registry and the quality of life prostate cancer cohort (QoL Prostate-MQ): challenges and prospects for reducing disparities in the Caribbean. BMJ Open 2018;8:e021540. doi:10.1136/ bmjopen-2018-021540

- Prepublication history for this paper is available online. To view these files, please visit the journal online (http://dx.doi. org/10.1136/bmjopen-2018021540).

Received 13 January 2018 Revised 1 June 2018 Accepted 15 June 2018

Check for updates

(c) Author(s) (or their employer(s)) 2018. Re-use permitted under CC BY-NC. No commercial re-use. See rights and permissions. Published by BMJ.

For numbered affiliations see end of article.

Correspondence to

Dr Clarisse Joachim;

clarisse.joachim@hotmail.fr

\section{ABSTRACT}

Purpose Recording cancer data in cancer registries is essential for producing reliable population-based data for service planning, monitoring and evaluation. Prostate cancer ( $\mathrm{PCa}$ ) remains the most frequent type of cancer in terms of incidence and mortality in men in the Caribbean. The quality of life PCa cohort will assess quality of life and patient outcomes in Martinique using a digital platform for patient-reported outcome measures.

Participants The Martinique Cancer Registry database is the largest clinical database among the French population-based cancer registries in the Caribbean, including more than 38000 cancer cases, with 1650 new cancer cases per year, including 550 new PCa cases per year (2010-2014 latest period). In 2018, follow-up will include vital status, assessment of quality of life with the European Organisation for Research and Treatment of Cancer Quality of Life Questionnaire (QLQ) Core 30 and the Prostate cancer module QLQ-PR25. Urinary incontinence and erectile dysfunction recorded prior to treatment will be analysed 1 and 5 years after treatment.

Findings to date The registry includes data on circumstances of diagnosis, clinical stage at diagnosis. For $\mathrm{PCa}$, the registry includes blood prostate-specific antigen level at the time of diagnosis, Gleason score and primary treatment.

Future plans Further studies will provide detailed data regarding the quality of diagnosis and management of patients with $\mathrm{PCa}$ in Martinique; analysing quality of care will be the next challenge. Quality of life and patient outcomes will be evaluated using a digital platform for patient-reported outcome measurement and electronic records.

\section{INTRODUCTION}

Cancer registries, through their mission to perform public health surveillance and research in oncology, contribute to the development of public health. Cancer is now a major public health problem in both

\section{Strengths and limitations of this study}

The purpose of this prostate cancer ( $\mathrm{PCa}$ ) cohort is to foster collaboration and maximise use of data in the Caribbean.

- This study will provide insights into the high rates of incidence and survival of $\mathrm{PCa}$ observed in Martinique, as compared with other Caribbean countries.

- The quality of life PCa cohort will be constituted in the context of an established, internationally recognised cancer registry, thus promoting high-quality data verification and recording.

- Data on clinical stage at diagnosis, blood prostate-specific antigen level at diagnosis, Gleason score, primary treatment, quality of life, urinary incontinence and erectile dysfunction prior to treatment will be analysed, where applicable, at 1 and 5 years after treatment.

- Potential limitations include the fact that comorbidities are not recorded and thus cannot be taken into account in statistical analyses, and potential loss to follow-up due to patients who travel abroad for therapy.

developed and transitioning countries. Prostate cancer (PCa) incidence and mortality rates are high in the Caribbean (respectively 79.8 and 29.0 cases per 100000 men); the French West Indies rank first for both PCa incidence and mortality rates. ${ }^{1}$ Over the 20102014 period, 4929 new cancer cases were diagnosed in men in Martinique, including 2676 cases of $\mathrm{PCa}$ ( $54.3 \%$ of cancers in men). This cancer, with a mean of 535 registered cases per year and a world-standardised incidence rate of 161.1 per 100000 and world-standardised mortality rate of 23.5 per 100000 , is the most common cancer in men 
in Martinique, with a higher incidence of PCa compared with data from other population-based cancer registries in the Caribbean region.

Health-related quality of life (QoL) is a multidimensional concept that encompasses physical, psychological and social aspects, as well as biological and clinical parameters. ${ }^{23}$ Consequently, diagnostic and therapeutic management for PCa can impact on patients' QoL in the short and long term, in view of the potential for adverse effects during and after therapy. In addition, there is also a risk of overdiagnosis and overtreatment for non-aggressive forms of disease that are diagnosed at very early stages, thereby increasing the number of patients whose QoL is affected. These potential risks must be taken into account when discussing goals of care with patients. ${ }^{45}$

Among the adverse effects of PCa treatments, chronic urinary problems and sexual dysfunction are often reported. Indeed, PCa therapies, particularly surgery and radiotherapy, can engender disorders of the urinary tract (eg, incontinence) or sexual symptoms (erectile dysfunction). ${ }^{6}$

Further research is needed to analyse the clinical management of cancer and to provide data for cancer surveillance and clinical research in the Caribbean. Risk factors such as genetic factors or environmental exposures may play an important role in the higher incidence observed in our region. ${ }^{78}$ Higher risks of PCa have been reported in African-Caribbean populations; and PCa was also the main cause of loss of disability-adjusted life-years in Latin America and the Caribbean. ${ }^{9-12}$ In the Caribbean, there is a compelling need for better information and higher quality data to improve cancer outcomes. The Martinique Cancer Registry (MCR) contributes to the implementation of public health studies to evaluate public health risks as a tool for epidemiological surveillance, and as a decision-making aid for local and national public health authorities since 1981. This registry is a member of the French Network of Cancer Registries (FRANCIM) and represents a major asset in terms of epidemiology for local and regional health authorities. The QoL PCa cohort is located in and conducted as part of the MCR.

The proposed study aims to reduce disparities in access to care and in outcomes, by bringing about improved PCa management and broader knowledge, supported by a digital platform for patient-reported outcomes. This project aims to pioneer the use of innovative technologies for the implementation of a QoL focused approach to cancer in the Caribbean.

There is also ongoing scientific collaboration for cancer surveillance across the Caribbean zone through the regional activities of cancer registries, including the French West Indies, the Caribbean and the International Association of Cancer Registries. The current project will promote QoL best practices in PCa and help public health agencies to prioritise QoL among the key indicators of health status in cancer survivors.
QoL has previously been investigated in several other PCa cohorts around the world. The CaPSURE (Cancer of the Prostate Strategic Urologic Research Endeavour) demonstrated associations between several QoL scores and survival using the Short Form 36-item in patients with localised PCa. ${ }^{13}$ The CEASAR (Comparative Effectiveness Analysis of Surgery and Radiation Study Results) study, performed in 2011-2012, assessed functional outcomes (sexual, incontinence, urinary domains) after prostatectomy or external beam radiotherapy and showed significant results according to the severity of PCa at diagnosis. ${ }^{14}$

The Prostate Cancer Outcomes Study, a population-based cohort from participating Surveillance, Epidemiology and End Results, also enrolled patients to assess differences in pretreatment urinary and sexual function in 1994-1995. ${ }^{15}$ A further study that investigated the factors associated with treatment decision regret underlined the importance of informed decision making in men treated by prostatectomy and the impact of side effects on QoL among long-term survivors of localised PCA. ${ }^{16}$

In France, a population-based case-control study was performed in 2011 (QALIPRO) among PCa survivors from 11 French cancer registries and reported that numerous urinary and sexual dysfunctions persisted up to 10 years after treatment. ${ }^{17}$

To date, no study has investigated QoL among patients with PCa in the Caribbean region, and data on PCa by cancer severity and functional outcomes are lacking. The epidemiological specificities (ie, genetic characteristics or predisposition, exposure to environmental risk factors), the management approaches in the island context as well as the severity profile of PCa in the Caribbean are features that we have in common with the other countries in the Caribbean region. Beyond data from other ongoing PCa cohorts, this study will additionally identify determinants of QoL and survival in a region that has the highest PCa incidence worldwide, using a digital platform to enhance partnership in the fight against cancer, and based on patient-reported outcome measures.

\section{Cancer registry description}

The MCR was created to enable exhaustive and continuous recording of all cases of cancer in persons living in Martinique (French West Indies in the Caribbean-390000 inhabitants) and to ensure privacy and confidentiality in line with guidelines for good practices. This registry has been participating in epidemiological surveillance and evaluation of cancer, through the analysis of incidence and mortality data over time including more than 38000 cases since 1981 . The MCR covers the whole urban $(98 \%)$ and rural $(2 \%)$ population of Martinique, thanks to exhaustive and continuous data collection for all cancer cases. Key indicators are calculated to study the trends in different types of cancers across the region of Martinique. The interval between diagnosis and case identification in the registry is about 2 years; this makes it possible to avoid accidental creation of duplicate records. In Martinique, there are no Death Certificate 
Only registrations, because reliable data regarding deaths for patients residing in Martinique are obtained from the French epidemiological centre on medical causes of death from the French National Institute of Health and Medical Research (CépiDc, Inserm: http://www.cepidc. inserm.fr/site4/), ensuring completeness of death information. Active follow-up of vital status is also performed and is based on medical records and administrative databases. Vital status updates and corrections of previous years are made continuously.

During the latest period (2010-2014), the mortality:incidence ratio was $<20 \%$. The percentage of PCa cases with morphological verification in 2013 was about $99 \%$. Potential threats to completeness of follow-up due to population mobility (including for treatment) represent $<5 \%$ of PCa cases, since we have access to treatment data for all French territories (mainland France plus all the overseas territories) through the national social security system.

A legal framework has been established for registration, confidentiality and encryption of data. The population is covered by the national health insurance system, plus optional private insurance. There is a legislative authority behind the registry, although cancer reporting is not obligatory in Martinique. The Martinique Cancer Registry database was approved.

In view of the high quality of the database of the MCR, the registry data are published regularly at an international scale through the International Agency for Research on Cancer (Cancer incidence in Five Continents ${ }^{18}$ ) since 1981 .

\section{Prostate cancer cohort description}

All incident patients with invasive cancer diagnosed in Martinique between 1 January 1981 and 31 December 2014 were recorded in the database of the registry in strict conformity with the international standards laid down by the International Agency for Research on Cancer, ${ }^{19}$ the FRANCIM network and the European Network of Cancer Registries.

This is the largest clinical database of the French population-based cancer registries in the Caribbean, including more than 38000 cancer cases, with 1650 new cancer cases per year including 550 new PCa cases per year (2010-2014 latest period). Taking into account a 5-year survival rate $>90 \%$ and a participation rate of almost $70 \%$, the anticipated size of the QoL prostate cohort is about 300 patients per year.

In 2018, QoL tools will be implemented as part of routine practice and documented in the patient's electronic medical records. Patients will be contacted 1 and 5 years after diagnosis to assess their QoL, which will be recorded in the electronic medical records.

Patients are included after diagnosis and followed up for a period of 5 years.

The primary objective of this cohort is to evaluate overall QoL, and QoL related to sexual dysfunction and urinary troubles among patients with PCa in Martinique for a period of 5 years after diagnosis.
Secondary objectives are:

- To evaluate overall survival at 5 years, and to identify prognostic factors for overall survival, taking the following variables into account: age, comorbidities, circumstances of diagnosis, tumour stage, type of treatment (surgery, radiotherapy, androgen deprivation therapy).

- To compare rates of urinary disorders and erectile dysfunction during the 5 years since diagnosis, according to the initial management strategy.

Thanks to data cross-matching and analysis of all available data sources, the MCR guarantees high-quality information about cancer in the region of Martinique. The registry is currently actively cooperating with a range of local organisations to ensure an exhaustive data collection circuit (discharge reports, laboratory results, pathology findings, people qualified as having chronic disease by the social security system, clinical patient file reviews...). Data are extracted anonymously using the International Classification of Diseases version 10 codes and the International Classification of Diseases for Oncology. ${ }^{20}$

The MCR database includes data on trends in incidence and mortality, as well as survival data for all cancer cases since the set-up of the registry in 1981. New variables have been added over time; primary treatments and disease characteristics at diagnosis were registered depending on tumour site recommendations. As of 2005, the following variables were recorded for PCa cases: circumstances of diagnosis, blood prostate-specific antigen (PSA) level at the time of diagnosis, Gleason score and primary treatment. Clinical stage at diagnosis was classified into localised (T1N0M0-T2N0M0) versus advanced (T3/T4 $\mathrm{n}+\mathrm{M}+$ ) based on the TNM classification, seventh edition (2010).

The D'Amico classification was used to stratify patients into three groups according to their risk of biological recurrence.

In a recent cohort study performed on PCa cases in Martinique, clinical stage at diagnosis was available for analysis in $85.2 \%$ patients. To avoid missing data, advanced disease (T4 or N1 or M1) will be checked, followed by evidence of locally advanced disease (T3) and then localised disease (T1 or T2) to get TNM stage. PSA and Gleason score were available in $100 \%$ patients, because it is mandatory to record these variables in medical records for the assessment of treatment strategy during multidisciplinary meetings.

In 2018, the MCR will start to assess QoL and patient outcomes in a dedicated research project using a digital platform for patient-reported outcome measures and electronic records. In the future, this platform for cancer surveillance could also enable QoL research projects, clinical research and genomics analysis, taking into account the level of development in information technology in the Caribbean.

We will record patterns of care among patients with PCa; treatments performed during the first 2 years were evaluated according to the clinical characteristics of the 
patients (age, clinical stage at diagnosis, Gleason score and D'Amico classification).

A previous analysis of different treatment combinations in PCa in our registry showed that almost $80 \%$ of patients received at least one treatment, while $20 \%$ had non-invasive treatment, active surveillance or watchful waiting.

According to medical health insurance data for Martinique, $95 \%$ of patients are treated in Martinique. For patients who seek care outside of Martinique (eg, mainland France, other French overseas territories), then the type of therapy received can be obtained from the French medical informatics system of the national social security system.

In 2018, urinary incontinence and erectile dysfunction will be analysed using validated questionnaires (ie, the International Index of Erectile Function (IIEF), ${ }^{21}$ and International Prostate Symptom Score (IPSS)) prior to treatment, and at 1 and 5 years after treatment. QoL will be measured using the European Organisation for Research and Treatment of Cancer Quality of Life Questionnaire Core $30^{22}$ (EORTC QLQ-C30) and the Prostate cancer module QLQ-PR25, ${ }^{23}$ once the patient has provided informed consent.

The urinary incontinence/erectile dysfunction domain explores how the urogenital effects of PCa impact on the patient's overall QoL (fatigue, weight loss, etc). These questionnaires have been validated internationally in their French language version, thus enabling comparison with other international studies. The questionnaires will be administered by the caregiving team, or self-completed by the patient via mailed surveys linked to a digital platform for data collection and quality control. This will ensure the exhaustiveness of the variables included in the database, by using data quality control programmes to verify that these data are collected systematically. In a recent study using EORTC QLQ-C30 and PR25, the response rate was $88 \%$ out of 120 patients contacted..$^{24}$ Our anticipated response rate is about $70 \%$.

\section{Statistical analysis}

First, in descriptive analysis, qualitative variables will be presented as number (percentage), and quantitative variables as mean $\pm \mathrm{SD}$, if normally distributed, or median (IQR) if non-normally distributed. Multivariate analysis will be performed to identify the factors associated with the primary outcome, by binary logistic or polytomic regression for qualitative variables, and using multivariate linear regression for quantitative variables. Results will be presented as OR with 95\% CI. The primary outcomes are: overall QoL in patients with cancer as assessed by the EORTC QLQ-C30, PCa-specific QoL as assessed by the EORTC-PR25, erectile function as assessed by the IIEF-5 and urinary function as assessed by the IPSS.

Univariate analysis will be performed to identify variables potentially associated with outcomes, by linear regression for QoL scores. Survival curves will be plotted using the Kaplan-Meier method. Candidate variables for inclusion in the multivariate models will be all those yielding a $\mathrm{p}$ value $<0.20$ by univariate analysis.

Multivariate analysis will be performed to identify the variables independently associated with the outcomes, namely multivariate linear regression for QoL scores, and a Cox proportional hazards model to estimate HR for death. One-year and 5-year survival will be evaluated to assess the statistical differences between survival curves for each categorical variable.

The main prognostic and therapeutic factors to be included in the model will be: age, PSA, D'Amico risk group, stage, type of treatment (surgery, radiotherapy, brachytherapy, watchful waiting) and the different scores obtained in the questionnaires.

All tests will be performed using SAS V.9.2 (SAS Institute, Cary, North Carolina, USA) and a p value $<0.05$ will be considered statistically significant.

\section{Patient and public involvement}

By definition, the primary outcome of this study, namely QoL in patients with PCa is directly informed by patients' priorities, experiences and preferences, insofar as we will measure how the disease impacts on their own perception of their QoL. Although the primary outcomes directly measure patient-reported outcomes, patients were not involved in the design of this study.

Regarding patient involvement, new prostate cases for QoL assessment are identified through multidisciplinary team meetings that are held jointly between public and private healthcare establishments, as well as through medical records and the registry, according to the registry procedures approved by the French National authority for the protection of privacy and personal data.

The French ethical regulations require that patients receive detailed oral and written information and have time for deliberation prior to signing a written informed consent. Potential participants for QoL measures are approached to provide informed consent after discussion of their case during joint public-private multidisciplinary team meetings, thus ensuring that all potential patients (ie, treated in both the public and private sectors) are reached. The treating physician invites the patient to participate, delivers information about the aims of the study and informs the patient that their personal medical data will be used for research purposes, and that they have a right to access, delete and/or modify their personal data at any time.

The results of the study will be disseminated to study participants by making the results available at the end of the study in the form of publications in scientific journals, and summaries for the lay public, which will be made available on the interactive digital platform, or on request from the registry.

\section{Findings to date}

To date, several reports have been published by the Martinique University Hospital Oncology department and the MCR, mainly relating to PCa, lung cancer and 


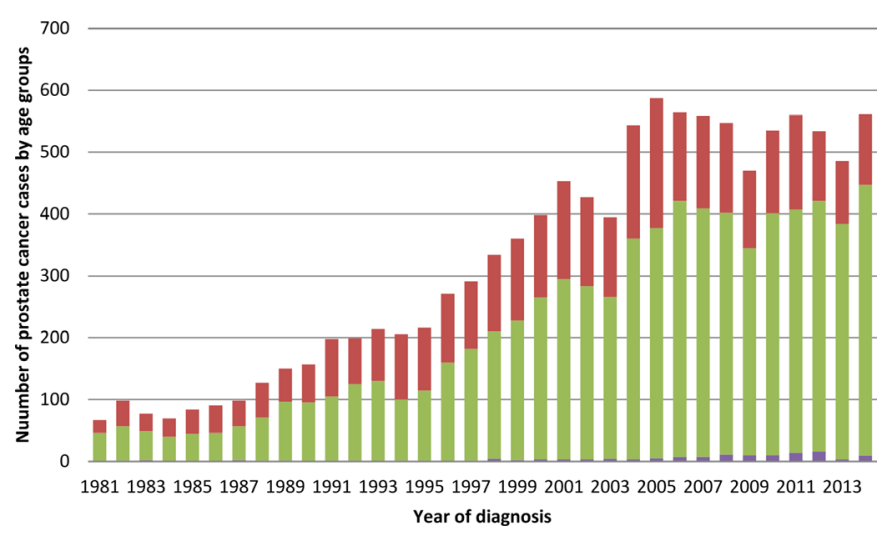

Figure 1 Number of patients with prostate cancer diagnosed by age group over the period 1981-2014, as per 2017 data from the Martinique Cancer Registry database. Blue bars represent patients aged $<50$ years, green bars represent patients aged 50 to 74 years and red bars represent patients aged 75 years and older at the time of diagnosis.

colorectal cancer. ${ }^{25-33}$ A first study on PSA kinetics after brachytherapy was retrospectively performed and showed that PSA bounce seemed earlier and was more intense. ${ }^{34}$ Another study in 370 patients with PCa reported biochemical outcomes of patients treated by brachytherapy from 2005 to 2014 in the University Hospital of Martinique. Major findings of this study included excellent rates of medium-term biochemical control in both low and selected intermediate-risk localised $\mathrm{PCa} .{ }^{35}$ In a high-resolution study on $\mathrm{PCa}$ in 452 patients, clinical stage at diagnosis was available in $385(85.2 \%)$; tumours were localised in $288(74.8 \%)$ and advanced in $97(25.2 \%)$. Overall, $18 \%$ were at low risk, $44.1 \%$ intermediate and $37.9 \%$ at high risk according to the D'Amico scale. This study provides detailed data regarding the quality of diagnosis and management of patients with $\mathrm{PCa}$ in Martinique. There was an increasing proportion of advanced tumours with increasing age, though this relation was not statistically significant. Conversely, we observed a statistically significant increase in Gleason score and D'Amico risk group for the oldest patients ( $\geq 65$ years). For example, among those aged 75 years and older, 52\% were in the highest risk group, as compared with only $29 \%$ among those younger than $65(p=0.0004)$. The diagnostic and therapeutic management was in line with international recommendations, except for positron emission tomography (PET)-CT imaging, which is not yet available in Martinique.

\section{Strengths and limitations}

This cohort has several strengths, but also some limitations that deserve to be underlined.

First, as outlined above, high data quality and near-total completeness at baseline are anticipated, thanks to the established and internationally validated practices of the existing registry. In addition, continuous cross-checking, and consistent analysis of multiple data sources, in line with national and international guidelines for registry data collection, enables the MCR to provide high-quality data on cancer in Martinique. The quality control procedures in place regarding implementation of cohort studies, access to data in medical files and coordination with healthcare professionals from the public and private sectors guarantee thorough data exhaustiveness and follow-up, from diagnosis through to the end of post-treatment follow-up. Treatment administered in-hospital can be identified from data sources already used in the registry (eg, medical informatics databases from the national social security system). However, we will not have access to data regarding treatment performed more than 2 years after diagnosis, and this represents a potential limitation. The use of the digital platform to record and analyse health data should help to boost participation rates and data completion and will guarantee high-quality control of the data thus collected.

We anticipate that there is minimal potential for loss to follow-up, since the MCR regularly updates data on vital status and can thus generate a list of patients to be contacted, according to the date of diagnosis as recorded in the registry. Patients attend regular consultations for the follow-up of their disease and for biological tests at any suspicion of recurrence and can be reminded at each visit of the importance of follow-up. This regular contact also makes it possible to administer the QoL questionnaires at regular intervals, and additional access for patients via the digital platform should help maintain completion rates near maximum. Finally, for patients who travel outside of Martinique for treatment, we can obtain data regarding treatment anywhere in France (eg, other overseas territories or in mainland France) from the social security system. Thus, thanks to the wide range of sources providing data to the registry, attrition is minimised.

\section{Future perspectives}

Research projects on PCa, colorectal cancer and haematological malignancies are needed in the Caribbean. The epidemiology of cancer in the French West Indies differs from that of mainland France, but also from that of other countries in the Caribbean and the Americas. Accordingly, PCa remains the most frequent type of cancer in terms of incidence and mortality in men. The observed incidence of this type of cancer in Martinique is almost twice as high as that in mainland France ${ }^{36} 37$ (figures 1-3). Since 2014, the registry has focused on producing scientific publications to meeting the compelling need for reliable and robust epidemiological data, in accordance with the objectives of collaboration and research within the Caribbean. Compared with previous years, a stronger will to enhance integration has been fostered within the MCR and has led to the creation and implementation of multicentre research projects, contributing to improved cohesion and cooperation in sharing of cancer data across the Caribbean zone.

It is anticipated that the results of this cohort study will be generalisable beyond Martinique. Indeed, despite the sociodemographic and health system diversity observed 


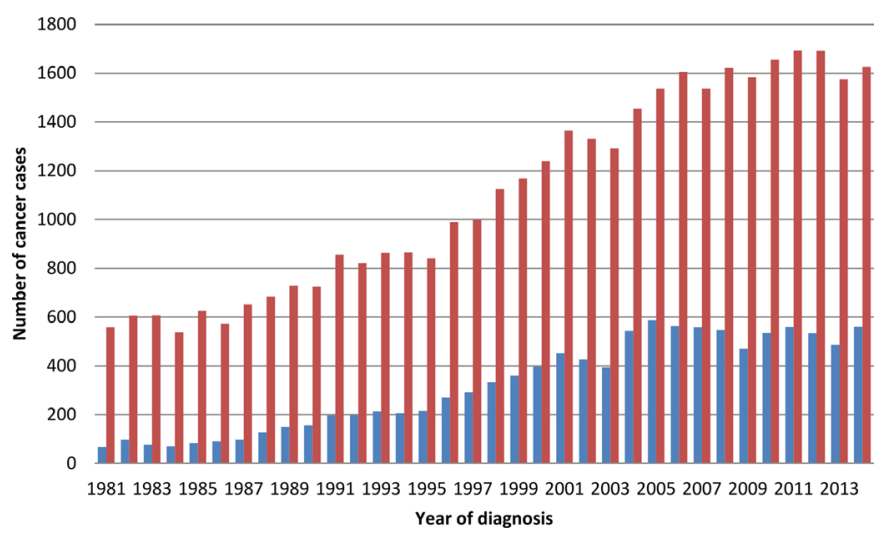

Figure 2 Number of cancer cases per year over the period 1981-2014, as per 2017 data from the Martinique Cancer Registry database. Red bars represent all cancers; blue bars represent prostate cancer.

across the Caribbean, the results of this study will make it possible to design QoL cohort studies for all patients with cancer throughout the region, using the EORTC questionnaires that are validated in French, Spanish and English language versions. Furthermore, this project could be extended in the future to other population-based cancer registries in the Caribbean, in view of the high incidence of this cancer in this region.

For the Caribbean zone, data are sparse, particular for countries that do not have a cancer registry and are based primarily on estimations and projections. Recording cancer data in cancer registries is essential for the production of reliable epidemiological data, and also contributes to improving management and reducing mortality. ${ }^{38}$ Countries with limited resources, such as island states in the Caribbean, have high mortality, yet do not all have population-based cancer registries to evaluate the extent of disease. It would therefore be beneficial if other existing registries could contribute to the production of international epidemiological statistics for this area. ${ }^{3840}$ A multicentre study on QoL in patients with PCa

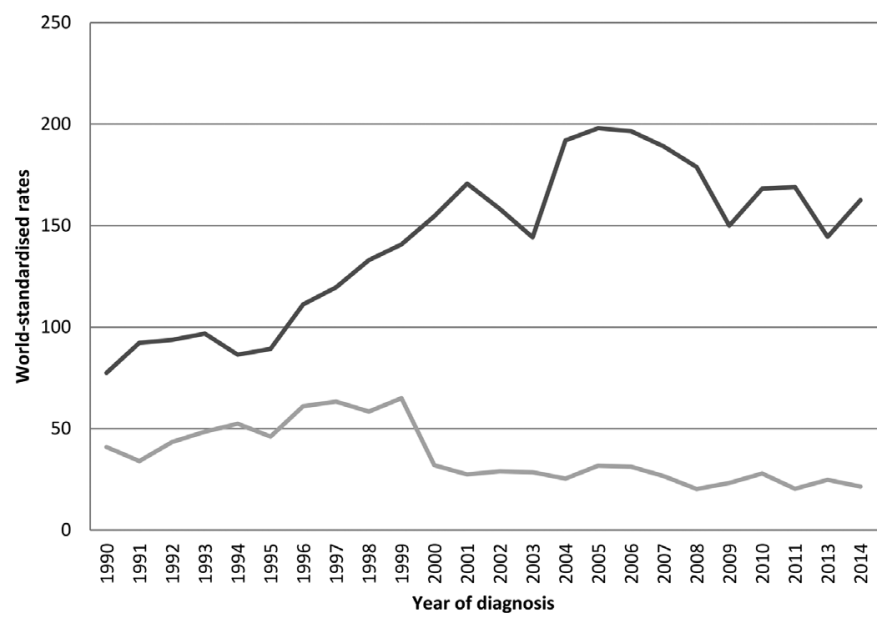

Figure 3 World-standardised incidence and mortality rates for prostate cancer in Martinique for the period 1981-2014 (excluding 2012). Dark grey line represents incidence; light grey line represents mortality. could be extended to the English-speaking countries of the Caribbean zone with population-based cancer registries and would be highly beneficial for epidemiological cancer surveillance in this geographical area. The findings may also help to identify disparities in such factors as access to care, outcomes according to sociodemographic or disease characteristics, thus focusing the attention of decision-makers and health authorities on the unmet needs for cancer care in these populations.

Finally, this project will initiate collaboration on $\mathrm{PCa}$ with existing or emerging registries in the Caribbean, with a view to participating in a pan-regional cancer surveillance system in this geographic zone in the future. New research programmes will also allow data collection on PET-CT imaging with development of dedicated isotopes in the future.

The results of this cohort will be an important impetus to bring about change. The expected repercussions include the following:

- Clear description of the regional public health issues in terms of management and QoL, on a wide geographical scale and in the island context.

- Participation in the dissemination throughout the French overseas territories of epidemiological data and innovative projects in the area of QoL.

- Development of surveillance mechanisms and improvement of knowledge on the health status of populations in the overseas territories.

Future plans, beyond this cohort, aim to continue this research work as a tool to inform policy, in the areas of population health and health services research. Indeed, improving QoL after treatment for cancer is at the heart of the national cancer plan for the period 2014-2019, and one of the main objectives is to improve QoL via access to supportive care. The over-riding aim is to make prevention and management of sequelae systematic, and also to roll out a preventive approach after a cancer diagnosis. Preserving QoL before and especially after treatment is the first step in supportive care. The benefits can be assessed in personal, professional, psychological and social terms.

Based on the results that emerge from the PCa cohort, we plan to develop QoL indicators, and engage in improving professional practice to implement routine QoL approaches across the cancer care spectrum.

The main aims of this approach would be to:

- Impact on regional health policy.

- Develop public health indicators that include a dimension pertaining to QoL, for the study of healthcare pathways and unmet needs, especially in terms of management of erectile and urinary dysfunction.

- Improve coordination between services such as functional rehabilitation for urinary disorders, and develop the field of oncosexuality in Martinique, with perspectives to cooperate on these topics with other disciplines.

- Create competent clinical teams with a view to improving healthcare pathways, including QoL, 
thanks to the systematic implementation of QoL assessments in routine practice.

\section{Author affiliations}

${ }^{1}$ UF1441 Registre des cancers de la Martinique, Pôle de Cancérologie Hématologie Urologie Pathologie, CHU Martinique, Fort-de-France, Martinique

${ }^{2}$ Groupe d'Étude, de Formation et de Recherche en Andrologie, Urologie et Sexologie Médecine de la Reproduction, Toulouse, France

${ }^{3}$ Groupe de recherche en fertilité humaine,, , CHU Toulouse Paule de Viguier, Toulouse, France

${ }^{4}$ Pôle de Cancérologie Hématologie Urologie Pathologie, CHU Martinique, Fort-deFrance, Martinique

${ }^{5}$ Pôle de Gériatrie, $\mathrm{CHU}$ de Martinique, Fort-de-France, Martinique

${ }^{6}$ Unité d'aide Méthodologique, Pôle Recherche et Santé publique, $\mathrm{CHU}$ de Reims, Reims, France

${ }^{7}$ EA 3797, Université de Reims Champagne-Ardenne, Faculté de Médecine, Reims, France

${ }^{8}$ Département de Médecine Interne et Gériatrie, CHU de Reims, Reims, France ${ }^{9}$ Service de Médecine nucléaire, Pole d'imagerie Médicale, CHU Martinique, Fortde-France, Martinique

Acknowledgements The authors thank FRANCIM Network, Santé Publique France, INCa, International Agency for Research on Cancer, patients and all those who contributed to the recording of cancer data in the registries: the Oncology Haematology Urology Pathology Division of the University Hospital of Martinique, the laboratories and departments of anatomy, cytology, and pathology; the departments of medical informatics of the public and private hospitals; the local offices of the national social security service; and general practitioners and specialists. We thank Fiona Ecarnot, MSc (EA3920, University Hospital Besancon, France) for editorial assistance.

Contributors CJ and JVB contributed to the concept of the study. CJ made major contributions to the conception, design of the work and the acquisition, analysis and interpretation of data for the work; drafted the work for important intellectual content; and gave final approval of the version to be published. CJ and $\mathrm{JM}$ managed the Martinique Cancer Registry Database and TA created the data collection tools. CJ, JM and TA made the statistical analysis plan of the Project. JVB, SUG, TA, VVH, LG, JLN, MD and KF contributed to the acquisition, analysis and interpretation of the data and revised the protocol critically for important intellectual content. VVH, JVB, TA and CJ are PI on the quality of life prostate cancer cohort. LG, $\mathrm{MD}$, and $\mathrm{JLN}$ are $\mathrm{PI}$ for prostate cancer management in the elderly of the Cohort. JVB, LG, MD, JLN, VVH, OPL, PE and TA revised the work for important intellectual content, and gave final approval of the version to be published. All authors have read and approved the final version of the manuscript to be published.

Funding This study was funded by the Martinique Cancer Registry.

Competing interests None declared.

Patient consent Not required.

Ethics approval The Martinique cancer registry database was approved by the French National authority for the protection of privacy and personal data (Commission Nationale Informatique et Libertés, CNIL Nº 987 001).

Provenance and peer review Not commissioned; externally peer reviewed.

Data sharing statement Requests to access the data are welcome and will be considered by the Scientific Committee of the MCR. For more information, please contact Clarisse Joachim, at Clarisse.joachim@chu-martinique.fr

Open access This is an open access article distributed in accordance with the Creative Commons Attribution Non Commercial (CC BY-NC 4.0) license, which permits others to distribute, remix, adapt, build upon this work non-commercially, and license their derivative works on different terms, provided the original work is properly cited, appropriate credit is given, any changes made indicated, and the use is non-commercial. See: http://creativecommons.org/licenses/by-nc/4.0/.

\section{REFERENCES}

1. Bray F, Piñeros M. Cancer patterns, trends and projections in Latin America and the Caribbean: a global context. Salud Publica Mex 2016;58:104-17.
2. Wilson IB, Cleary PD. Linking clinical variables with health-related quality of life. A conceptual model of patient outcomes. JAMA 1995;273:59-65.

3. Revicki DA, Osoba D, Fairclough D, et al. Recommendations on health-related quality of life research to support labeling and promotional claims in the United States. Qual Life Res 2000;9:887-900

4. Andriole GL, Crawford ED, Grubb RL, et al. Prostate cancer screening in the randomized Prostate, Lung, Colorectal, and Ovarian Cancer Screening Trial: mortality results after 13 years of follow-up. $J$ Natl Cancer Inst 2012;104:125-32.

5. Wilt TJ, Brawer MK, Jones KM, et al. Radical prostatectomy versus observation for localized prostate cancer. N Engl J Med 2012;367:203-13.

6. Vij A, Kowalkowski MA, Hart T, et al. Symptom management strategies for men with early-stage prostate cancer: results from the Prostate Cancer Patient Education Program (PC PEP). J Cancer Educ 2013;28:755-61.

7. Cancel-Tassin G, Romana M, Gaffory C, et al. Region 2 of $8 q 24$ is associated with the risk of aggressive prostate cancer in Caribbean men of African descent from Guadeloupe (French West Indies). Asian $J$ Androl 2015;17:117-9.

8. Emeville $\mathrm{E}$, Broquère $\mathrm{C}$, Brureau $\mathrm{L}$, et al. Copy number variation of GSTT1 and GSTM1 and the risk of prostate cancer in a Caribbean population of African descent. PLoS One 2014;9:e107275.

9. Soerjomataram I, Lortet-Tieulent J, Parkin DM, et al. Global burden of cancer in 2008: a systematic analysis of disability-adjusted lifeyears in 12 world regions. Lancet 2012;380:1840-50.

10. Odedina FT, Akinremi TO, Chinegwundoh F, et al. Prostate cancer disparities in Black men of African descent: a comparative literature review of prostate cancer burden among Black men in the United States, Caribbean, United Kingdom, and West Africa. Infect Agent Cancer 2009;4(Suppl 1):S2.

11. Bouchardy C, Mirra AP, Khlat M, et al. Ethnicity and cancer risk in São Paulo, Brazil. Cancer Epidemiol Biomarkers Prev 1991;1:21-7.

12. Best Plummer WS, Persaud P, Layne PJ. Ethnicity and cancer in Guyana, South America. Infect Agent Cancer 2009;4(Suppl 1):S7.

13. Punnen S, Cowan JE, Chan JM, et al. Long-term health-related quality of life after primary treatment for localized prostate cancer: results from the CaPSURE registry. Eur Urol 2015;68:600-8.

14. Tyson MD, Koyama T, Lee D, et al. Effect of Prostate Cancer Severity on Functional Outcomes After Localized Treatment: Comparative Effectiveness Analysis of Surgery and Radiation Study Results. Eur Urol 2018;74:26-33.

15. Resnick MJ, Barocas DA, Morgans AK, et al. The Evolution of Self-Reported Urinary and Sexual Dysfunction over the Last Two Decades: Implications for Comparative Effectiveness Research. Eur Urol 2015;67:1019-25.

16. Hoffman RM, Lo M, Clark JA, et al. Treatment Decision Regret Among Long-Term Survivors of Localized Prostate Cancer: Results From the Prostate Cancer Outcomes Study. J Clin Oncol 2017;35:2306-14.

17. Kerleau C, Guizard AV, Daubisse-Marliac L, et al. Long-term quality of life among localised prostate cancer survivors: QALIPRO population-based study. Eur J Cancer 2016;63:143-53.

18. Forman FB, Kohler M, Piñeros E, et al. 164 ISPN.

19. Globocan I. Fact Sheets by Cancer. Secondary Fact Sheets by Cancer. http://globocan.iarc.fr/Pages/fact_sheets_cancer.aspx.

20. Fritz A. International classification of diseases for oncology: ICD-O. 3rd ed: World Health Organization, 2000.

21. Rosen RC, Riley A, Wagner G, et al. The international index of erectile function (IIEF): a multidimensional scale for assessment of erectile dysfunction. Urology 1997;49:822-30.

22. Fayers P, Bottomley A. EORTC Quality of Life GroupQuality of Life Unit. Quality of life research within the EORTC-the EORTC QLQ-C30. European Organisation for Research and Treatment of Cancer. Eur J Cancer 2002;38(Suppl 4):S125-33.

23. van Andel G, Bottomley A, Fosså SD, et al. An international field study of the EORTC QLQ-PR25: a questionnaire for assessing the health-related quality of life of patients with prostate cancer. Eur $J$ Cancer 2008;44:2418-24.

24. Buergy D, Schneiberg V, Schaefer J, et al. Quality of life after low-dose rate-brachytherapy for prostate carcinoma - long-term results and literature review on QLQ-C30 and QLQ-PR25 results in published brachytherapy series. Health Qual Life Outcomes 2018;16:21.

25. Veronique-Baudin J, Dieye M, Kouyoumdjian JC, et al. [Case-control study of the genes of receptors of the androgens of vitamin-D and of 5-alphareductase in a population of Afro-Caribbean population with prostate cancer]. Prog Urol 2006;16:303-10. 
26. Decastel M, Ossondo M, Andrea AM, et al. Colorectal cancer in patients seen at the teaching hospitals of Guadeloupe and Martinique: discrepancies, similarities in clinicopathological features, and p53 status. BMC Clin Pathol 2014;14:12.

27. Melan K, Volumenie JL, Wan-Ajouhu G, et al. Pregnancy-associatedcancer in the French West Indies (Martinique): maternal and neonatal outcomes. BMC Pregnancy Childbirth 2017;17:334.

28. Melan K, Janky E, Macni J, et al. Epidemiology and survival of cervical cancer in the French West-Indies: data from the Martinique Cancer Registry (2002-2011). Glob Health Action 2017;10:1337341.

29. Molinie V, Clément C, Peneau M, et al. [Not Available]. Prog Urol 2015;25:757-8.

30. Joachim C, Véronique-Baudin J, Razanakaivo M, et al. Trends in colorectal cancer in the Caribbean: A population-based study in Martinique, 1982-2011. Rev Epidemiol Sante Publique 2017;65:181-8.

31. Joachim C, Godaert L, Dramé M, et al. Overall survival in elderly patients with colorectal cancer: A population-based study in the Caribbean. Cancer Epidemiol 2017;48:85-91.

32. Dieye M, Véronique-Baudin J, Macni J, et al. L'incidence et la Mortalité par Cancer en Martinique entre 2006 et 2010. Données du registre général des cancers de la Martinique en Martinique entre 2006 et 2010. Leeds: AMREC, 2013.
33. Dieye M, Banydeen R, Macni J, et al. Geographic variations and temporal trends in prostate cancer in Martinique over a 25-year period. BMC Res Notes 2014;7:262.

34. Leduc N, Atallah V, Creoff $M$, et al. Prostate-specific antigen bounce after curative brachytherapy for early-stage prostate cancer: A study of 274 African-Caribbean patients. Brachytherapy 2015;14:826-33.

35. Atallah V, Leduc N, Creoff M, et al. Curative brachytherapy for prostate cancer in African-Caribbean patients: A retrospective analysis of 370 consecutive cases. Brachytherapy 2017;16:342-7.

36. INCa. Plan Cancer 2014-2019. Secondary Plan Cancer 2014-2019. 2014 http://www.e-cancer.fr/Expertises-et-publications/Cataloguedes-publications/Plan-cancer-2014-2019.

37. INCa. Les cancers en France, Les Données, INCa, édition 2015. Secondary Les cancers en France, Les Données, INCa, édition 2015. $2016 \mathrm{http} / / / w w w . e-c a n c e r . f r / E x p e r t i s e s-e t-p u b l i c a t i o n s / C a t a l o g u e-$ des-publications/Les-cancers-en-France-Edition-2015.

38. Gandaglia G, Bray F, Cooperberg MR, et al. Prostate cancer registries: current status and future directions. Eur Urol 2016;69:998-1012.

39. Van Hemelrijck M, Wigertz A, Sandin F, et al. Cohort Profile: the National Prostate Cancer Register of Sweden and Prostate Cancer data Base Sweden 2.0. Int J Epidemiol 2013;42:956-67.

40. Curado MP, de Souza DL. Cancer burden in Latin America and the Caribbean. Ann Glob Health 2014;80:370-7. 